\title{
Caracterização dos trabalhadores da enfermagem afastados por distúrbios osteomusculares em hospital universitário
}

Characterization of nurse staffing who are away from work due musculoskeletal disorders in a university hospital

Caracterización de trabajadores de enfermería en licencia por trastornos musculoesqueléticos en un hospital universitário

\section{Yuri Marques Souza', Daiane Dal Pai ${ }^{\mathrm{II}}$, Larissa Martini Junqueira ${ }^{\mathrm{III}}$, Andréia Barcellos Teixeira Macedo ${ }^{\mathrm{IV}}$, Juliana Petri Tavares ${ }^{\mathrm{v}}$, Eunice Beatriz Martin Chaves ${ }^{\mathrm{VI}}$}

Resumo: Objetivo: descrever características sociodemográficas e ocupacionais de trabalhadores da equipe de enfermagem afastados por distúrbios osteomusculares em hospital universitário e sua associação com o tempo de afastamento. Método: estudo quantitativo retrospectivo, transversal e analítico. Os dados foram coletados nos prontuários de trabalhadores afastados no período de 2012 a 2017, e analisados por meio da estatística descritiva e analítica. Resultados: de 2.761 afastamentos registrados, 449 se referiam a distúrbios osteomusculares (16,26\%), o maior percentual por dorsalgia $(41,5 \%)$ e 78 trabalhadores apresentaram diagnóstico psiquiátrico, principalmente depressão (43,4\%). Identificou-se no grupo com maior tempo de afastamento ( $>$ de 15 dias) o predomínio da categoria de auxiliares e técnicos de enfermagem $(\mathrm{p}=0,006)$, trabalhadores com menor mediana de idade $(\mathrm{p}=0,021)$ e de maior escolaridade $(\mathrm{p}=0,035)$. Conclusão: o elevado número de afastamentos por distúrbios osteomusculares, por vezes associado a outras comorbidades, exige acompanhamento e medidas preventivas nos locais de trabalho.

Descritores: Dor musculoesquelética; Transtornos traumáticos cumulativos; Saúde do Trabalhador; Licença médica; Enfermagem

\footnotetext{
I Enfermeiro, Graduação em Enfermagem, Universidade Federal do Rio Grande do Sul, Porto Alegre, Rio Grande do Sul, Brasil. yurims43@hotmail.com; https://orcid.org/0000-0001-7684-4582

II Enfermeira, Doutora em Enfermagem, Universidade Federal do Rio Grande do Sul, Porto Alegre, Rio Grande do Sul, Brasil. daiane.dalpai@gmail.com; https://orcid.org/0000-0002-6761-0415

III Acadêmica de Enfermagem, Graduanda de enfermagem, Universidade Federal do Rio Grande do Sul, Porto Alegre, Rio Grande do Sul, Brasil. larissamartinijunqueira@gmail.com; https://orcid.org/0000-0001-5849-163X

IV Enfermeira, Doutoranda de Enfermagem, Universidade Federal do Rio Grande do Sul, Porto Alegre, Rio Grande do Sul, Brasil. abtmacedo@gmail.com; https://orcid.org/0000-0003-4219-4731

V Enfermeira, Doutora em Enfermagem, Universidade Federal do Rio Grande do Sul, Porto Alegre, Rio Grande do Sul, Brasil. jupetritavares@gmail.com; https://orcid.org/0000-0003-4121-645X

vi Médica, Doutora em Clínica Médica, Universidade Federal do Rio Grande do Sul, Porto Alegre, Rio Grande do Sul, Brasil. ebmchaves@gmail.com; https://orcid.org/0000-0002-5129-8981
} 


\begin{abstract}
Aim: to describe the sociodemographic and occupational characteristics of nurse staffing from an university hospital who are away from work due to musculoskeletal cumulative disorders and the association with absenteeism. Method: retrospective and cross-sectional study. Were analysed medical records from 2012 to 2017, and analyzed using descriptive and analytical statistics. Results: Out of 2,761 recorded absences from nurse staffing, 449 referred to musculoskeletal disorders (16.26\%), the highest percentage due to back pain (41.5\%) and 78 workers had psychiatric diagnosis, especially depression (43.4\%). It was identified in the group with longer time of absence (\&gt; 15 days) the predominance of the category of nursing assistants and technicians $(\mathrm{p}=0.006)$, with lower median age $(\mathrm{p}=$ $0.021)$ and higher education $(p=0.035)$. Conclusion: The elevated number of absence due to musculoskeletal disorders, sometimes associated with other comorbidities, requires follow-up and prevention in the workplace.
\end{abstract}

Descriptors: Musculoskeletal Pain; Cumulative Trauma Disorders; Occupational Health; Sick Leave; Nursing

Resumen: Objetivo: Describir las características sociodemográficas y ocupacionales de los trabajadores del personal de enfermería en licencia debido a trastornos musculoesqueléticos en un hospital universitario y su asociación con el tiempo de ausencia. Método: estudio retrospectivo, transversal y analítico. Se analizaron los registros médicos de los trabajadores con licencia de 2012 a 2017, utilizando estadísticas descriptivas y analíticas. Resultados: De 2,761 ausencias registradas, 449 fueron trastornos musculoesqueléticos (16.26\%), debido a dolor de espalda (41.5\%) y 78 trabajadores tuvieron diagnóstico psiquiátrico, especialmente depresión (43.4\%). Se identificó en el grupo con mayor tiempo de ausencia (\&gt; 15 días) el predominio de la categoría de auxiliares y técnicos de enfermería $(\mathrm{p}=0.006)$, trabajadores con menor edad media $(\mathrm{p}=0.021)$ y educación superior $(\mathrm{p}=0,035)$. Conclusión: El alto número de ausencia por enfermedad debido a trastornos musculoesqueléticos, a veces asociados con otras comorbilidades, requiere seguimiento y prevención en el entorno laboral.

Descriptores: Dolor Musculoesquelético; Trastornos de Traumas Acumulados; Salud Laboral; Ausencia por Enfermedad; Enfermería

\title{
Introdução
}

$\mathrm{Na}$ enfermagem os profissionais são expostos a riscos para a sua saúde devido à realização de atividades que envolvem o trabalho estático e dinâmico no que tange ao sistema musculoesquelético. O trabalho estático é uma ação que exige contração contínua de alguns músculos para manter determinada posição. Já o trabalho dinâmico é aquele que permite contração e relaxamento alternados dos músculos. ${ }^{1}$

Revisão sistemática que avaliou lesões por esforço repetitivo na enfermagem demonstrou que os profissionais se tornam vulneráveis aos diversos tipos de distúrbios osteomusculares devido às condições e alta carga de trabalho. Outros fatores, como horas extras de trabalho, ritmo acelerado, ausência de pessoal, fragmentação de tarefas, força física excessiva, posturas inadequadas, carregar um peso maior que $10 \mathrm{~kg}$ e exercer carga horária maior que oito horas 
3 | Souza YMN, Dal Pai D, Junqueira LM, Macedo ABT, Tavares JT, Chaves EBM

diárias também estão ligados ao surgimento de distúrbios osteomusculares na enfermagem. ${ }^{2-4}$ Quanto ao conhecimento de enfermeiros sobre a prevenção de Distúrbios Osteomusculares Relacionados ao Trabalho (DORT) evidenciou-se que os principais fatores relacionados pelos profissionais ao surgimento de tais distúrbios foram a repetitividade de movimentos, a postura inadequada, o esforço físico excessivo e a jornada de trabalho prolongada. ${ }^{5}$

Os DORT são distúrbios crônicos nas estruturas musculoesqueléticas, causadas pelas atividades realizadas no trabalho. DORT pode ser considerado nome genérico dado a um conjunto de afecções multifatoriais que acometem músculos, tendões sinoviais, articulações, vasos e nervos, sendo um diagnóstico frequente entre trabalhadores de enfermagem. ${ }^{6-7}$

O contexto da prática de enfermagem está sendo estudada mundialmente e comparado com outras funções quanto às consequências à saúde dos trabalhadores. Pesquisa com objetivo de verificar fatores específicos do trabalho que influenciam na prevalência de dor musculoesquelética entre trabalhadores que exercem sua função no escritório, cuidadores e enfermeiros, evidenciou-se que 73,9\% dos enfermeiros ( $\mathrm{n}=221)$ referiram dor na região lombar, sendo percentual superior às demais ocupações. ${ }^{8}$ Já em outro estudo, sobre dor lombar no último ano, foram avaliadas enfermeiras, professoras e sonografistas, sendo que as primeiras $(\mathrm{n}=925)$ representaram o grupo mais acometido $(51 \%) .{ }^{9}$

Pesquisa desenvolvida no Irã, que objetivou buscar a relação da prevalência e o risco de distúrbios musculoesqueléticos da equipe de enfermagem no trabalho manual com o paciente, também corrobora os dados já apresentados ao mostrar que dentre 175 enfermeiros, 79,5\% apresentam algum distúrbio musculoesquelético, tendo destaque para a região lombar $(69,1 \%) .{ }^{10}$ $\mathrm{Na}$ Malásia, investigação realizada sobre prevalência e impacto dos distúrbios musculoesqueléticos relacionados ao trabalho entre 376 enfermeiras, verificou que, no ano de 2016, a taxa de absenteísmo devido a esses distúrbios aumentou de $6,2 \%$ para $18 \% .^{11}$ 
Estudo brasileiro que avaliou 1.574 atestados médicos de todos os trabalhadores da equipe de enfermagem em um hospital universitário mostrou que a maioria (19,7\%) dos atestados eram provenientes de doenças do sistema osteomuscular, dentre elas em destaque a Dorsalgia. ${ }^{12}$ Em uma revisão integrativa que investigou a relação do absenteísmo nas equipes de enfermagem, foram vistos treze artigos e dentre estes seis mostraram as doenças osteomusculares como sendo a doença predominante e causadora de afastamentos, evidenciando uma forte associação de doenças osteomusculares com o absenteísmo. ${ }^{13}$

Com base no exposto, a justificativa para esta investigação se baseia na importância de produzir e agregar conhecimento sobre o tema para embasar atividades de prevenção e promoção da saúde dos trabalhadores de enfermagem, assim como para o gerenciamento dos serviços de saúde, considerando o representativo impacto da população de enfermagem para a gestão de recursos e processos em hospitais.

A pesquisa desenvolvida teve como questão: Como se caracterizam os trabalhadores da equipe de enfermagem afastados por distúrbios osteomusculares em um hospital universitário? O objetivo foi descrever características sociodemográficas e ocupacionais de trabalhadores da equipe de enfermagem afastados por distúrbios osteomusculares em hospital universitário e sua associação com o tempo de afastamento.

\section{Método}

Estudo quantitativo transversal, retrospectivo e analítico. Realizado no Hospital de Clínicas de Porto Alegre (HCPA), uma instituição pública e universitária, integrante da rede de hospitais universitários do Ministério da Educação e vinculada academicamente à Universidade Federal do Rio Grande do Sul (UFRGS). A instituição conta com 842 leitos e possui uma equipe de enfermagem composta por 660 enfermeiros, 1521 técnicos de enfermagem e 477 auxiliares de enfermagem. 
5 | Souza YMN, Dal Pai D, Junqueira LM, Macedo ABT, Tavares JT, Chaves EBM

A amostra foi composta pelos prontuários dos profissionais da enfermagem $(\mathrm{n}=2.212)$ no período de 2012 a 2017, que consultaram no Serviço de Medicina Ocupacional (SMO) do HCPA. Foram incluídos registros dos trabalhadores de enfermagem afastados por motivo de doença e que fossem ser auxiliar, técnico de enfermagem ou enfermeiro e ter se afastado no período selecionado com algum dos Códigos Internacional de Doenças (CID’s): 25.5 - Dor Articular; 50 - Transtornos Discos Cervicais; 50.1 - Transtornos Disco Cervical com Radiculopatia; 50.2 - Outro Deslocamento de Disco Cervical;50.9 - Transtorno Não Especificado de Disco Cervical; 51 - Outros transtornos de Discos Intervertebrais; 51.0 - Transtornos de Discos Lombares e de Outros Discos Intervertebrais com Mielopatia; 51.1 - Transtornos de discos lombares e de outros discos intervertebrais com radiculopatia; 51.3 - Outra Degeneração Especificada de Disco Intervertebral; 51.9 - Transtorno Não Especif. de Disco Intervertebral; 53.1 - Síndrome Cervicobraquial; 54 - Dorsalgia ;54.1 - Radiculopatia; 54.2 - Cervicalgia; 54.3 - Ciatica; 54.4 - Lumbago com Ciatica; 54.5 - Dor Lombar Baixa; 54.9 Dorsalgia não Especificada; 65 - Sinovite e Tenossinovite; 65.0 - Abscesso da Bainha Tendínea; 65.2 Tendinite Calcificada; 65.3- Dedo em Gatilho; 65.4 - Tenossinovite Estiloide Radial de Quervain; 65.8 - Outras Sinovites e Tenossinovites; 65.9 - Sinovite e Tenossinovite Não Especificada; 66 - Ruptura Espontânea de Sinovia e de Tendão; 66.0 - Ruptura de Cisto Poplíteo; 66.5 - Ruptura Espontânea de Tendões Não Especificados; 67.8 - Outros Transtornos Especificados da Sinovia e do Tendão; 71.3 Outros Cistos de Bolsa Sinovial; 72.2 - Fibromatose da Fáscia Plantar; 72.5 - Fascite Não Classificada em Outra Parte; 75 - Lesões do Ombro; 75.0 - Capsulite Adesiva do Ombro; 75.1 - Síndrome do Manguito Rotador; 75.2 - Tendinite Bicepital; 75.3 - Tendinite Calcificante do Ombro; 75.5 - Bursite do Ombro; 75.8 - Outras Lesões do Ombro; 75.9 - Lesão Não Especificada do Ombro; 77.0 Epicondilite Medial; 77.1 - Epicondilite Lateral; 77.3 - Esporão do Calcâneo; 77.8 - Outras Entesopatias Não Classificadas em Outra Parte; 77.9 - Entesopatia Não Especificada no período de 2012 a 2017. Foram excluídos da amostra os afastamentos por licença gestação ou licença especial, bem como ausências ou incoerências no preenchimento das informações. 
A coleta de dados ocorreu por meio da solicitação de uma query (processo de extração de informações de um banco de dados no sistema eletrônico) dos prontuários de trabalhadores afastados no período de $1^{\circ}$ de julho de 2012 à $1^{\circ}$ de julho de 2017.

As variáveis de interesse que compuseram um banco de dados em planilha Excel foram: sexo, idade, estado civil, escolaridade, ocupação, anos de trabalho na instituição, índice de massa corporal (IMC), peso, altura, doenças crônicas, diagnóstico associado, número de afastamentos e total de dias de afastamento. Os dados foram submetidos à estatística descritiva e analítica com auxílio do SPSS versão 18.0. As variáveis qualitativas foram apresentadas por meio de frequência absoluta e relativa e a variáveis quantitativas por medidas de tendência central e dispersão.

Empregou-se estatística analítica para verificar diferenças entre os trabalhadores afastados com maior ou menor tempo, utilizando Qui-quadrado de Pearson e Exato de Fisher em análises de associação para variáveis categóricas, teste Mann Whitney para variáveis contínuas conforme assimetria da distribuição identificada pelo teste de normalidade de Shapiro-Wilk. Foram considerados significativos valores de $\mathrm{p}<0,05$.

Este estudo foi apreciado pelo Comitê de Ética em Pesquisa do HCPA em 26 de janeiro de 2018 sob parecer de número 2.474.947. Foram respeitados os aspectos éticos, conforme Resolução 466/2012 do Conselho Nacional de Saúde. Também foi utilizado o Termo de Autorização para Uso de Dados, sendo garantido o anonimato dos profissionais e respectivos prontuários na apresentação dos achados.

\section{Resultados}

Analisou-se o prontuário de 2.212 profissionais da enfermagem (auxiliares, técnicos de enfermagem e enfermeiros) que consultaram o SMO e constatou-se que $220(9,94 \%)$ haviam se afastado por distúrbios osteomusculares. Foram identificados 2.761 afastamentos, dentre os 
7 | Souza YMN, Dal Pai D, Junqueira LM, Macedo ABT, Tavares JT, Chaves EBM

quais $449(16,26 \%)$ se tratava de afastamentos por distúrbios osteomusculares. O serviço que mais apresentou afastamentos foi de internação clínica.

A Tabela 1 apresenta as características sociodemográficos e ocupacionais dos trabalhadores afastados por distúrbios osteomusculares.

Tabela 1 - Distribuição dos indivíduos afastados por distúrbios osteomusculares segundo dados sociodemográficos e ocupacionais. Porto Alegre, RS. 2018.

\begin{tabular}{|c|c|}
\hline Variáveis & $\mathbf{N}=\mathbf{2 2 0}$ \\
\hline \multicolumn{2}{|l|}{$\operatorname{Sexo}^{*}(n=219)$} \\
\hline Feminino & $188(85,8)$ \\
\hline Masculino & $31(14,2)$ \\
\hline Idade" $(\mathbf{n}=\mathbf{2 2 0})$ & $48(28-69)$ \\
\hline \multicolumn{2}{|l|}{ Estado Civil $^{*}(\mathbf{n}=219)$} \\
\hline Com Companheiro & $75(34,2)$ \\
\hline Sem Companheiro & $144(65,8)$ \\
\hline \multicolumn{2}{|l|}{ Escolaridade $^{*}(\mathbf{n}=\mathbf{2 1 9})$} \\
\hline >Ensino Médio & $194(88,6)$ \\
\hline$<$ Ensino Médio & $25(11,4)$ \\
\hline \multicolumn{2}{|l|}{ Ocupação* $(n=219)$} \\
\hline Téc. e Aux. de Enfermagem & $200(91,3)$ \\
\hline Enfermeiro & $19(8,7)$ \\
\hline Número de Afastamentos" $(\mathrm{n}=\mathbf{2 2 0})$ & $2,04(1-9)$ \\
\hline Total de Dias Afastados” $(n=220)$ & $120,99(4-1630)$ \\
\hline Anos de Trabalho na Instituição” $(\mathbf{n}=\mathbf{2 2 0})$ & $14(8-22)$ \\
\hline IMC” $(\mathbf{n}=\mathbf{2 2 0})$ & $27,90(19,14-51,20)$ \\
\hline \multicolumn{2}{|l|}{ Doença Crônica* $^{*}(\mathbf{n}=204)$} \\
\hline $\operatorname{Sim}$ & $144(70,6)$ \\
\hline Não & $60(29,4)$ \\
\hline \multicolumn{2}{|l|}{ Número de Doenças Crônicas* $(\mathbf{n}=\mathbf{2 2 0})$} \\
\hline Não & $75(34)$ \\
\hline Uma & $108(49)$ \\
\hline Duas ou mais & $37(17)$ \\
\hline \multicolumn{2}{|l|}{ Diagnóstico Psiquiátrico* $(n=196)$} \\
\hline $\operatorname{Sim}$ & $78(39,8)$ \\
\hline Não & $118(60,2)$ \\
\hline \multicolumn{2}{|l|}{ Tipo de Diagnóstico Psiquiátrico* $(n=76)$} \\
\hline Depressão & $33(43,4)$ \\
\hline Ansiedade & $19(25)$ \\
\hline Transtorno Bipolar & $8(10,5)$ \\
\hline Outros & $16(21)$ \\
\hline
\end{tabular}

Notas: *n (\%);"mediana (intervalos interquartílicos).

Fonte: banco de dados dos autores, 2018 
Com o intuito de relacionar os dados apresentados com os afastamentos de longa e curta duração foi realizada análise de distribuição das características sociodemográficas e laborais entre os grupos, o que permite pormenorizar a análise da caracterização pretendida no presente estudo (Tabela 2).

Tabela 2 - Distribuição dos trabalhadores de enfermagem afastados com menos de 15 dias e mais de 16 dias segundo dados sociodemográficos e ocupacionais. Porto Alegre, RS. 2018.

\begin{tabular}{|c|c|c|c|}
\hline Variáveis & $\leq 15$ dias & $>15$ dias & $p$ \\
\hline \multicolumn{4}{|l|}{ Sexo* } \\
\hline Feminino & $7(3,8)$ & $179(96,2)$ & \multirow{2}{*}{0,597} \\
\hline Masculino & $0(0)$ & $30(100)$ & \\
\hline \multicolumn{4}{|l|}{ Escolaridade* $^{*}$} \\
\hline 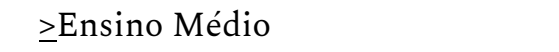 & $4(2,1)$ & $187(97,9)$ & \multirow{2}{*}{$\mathbf{0 , 0 3 5}$} \\
\hline$<$ Ensino Médio & $3(12)$ & $22(88)$ & \\
\hline \multicolumn{4}{|l|}{ Estado Civil $^{*}$} \\
\hline Com Companheiro & $4(2,8)$ & $137(97,2)$ & \multirow{2}{*}{0,696} \\
\hline Sem Companheiro & $3(4)$ & $72(96)$ & \\
\hline Idade“ & $58(52-59)$ & $48(41-53)$ & 0,006 \\
\hline Peso“ & $66(60-73)$ & $73,2(65-86,5)$ & 0,115 \\
\hline Altura“ & $1,61(1,57-1,64)$ & $1,63(1,59-1,7)$ & 0,207 \\
\hline IMC“ & $26,1(23,4-27,2)$ & $27,33(24,3-30,8)$ & 0,216 \\
\hline \multicolumn{4}{|l|}{ Ocupação* } \\
\hline Aux./Téc. Enf. & $4(2)$ & $193(98)$ & \multirow{2}{*}{$\mathbf{0 , 0 1 6}$} \\
\hline Enfermeiro (a) & $3(15,8)$ & $16(84,2)$ & \\
\hline Anos de Trabalho na Instituição" & $22(8-29)$ & $14(7,5-21)$ & 0,139 \\
\hline \multicolumn{4}{|l|}{ Doença Crônica* } \\
\hline Sim & $6(4,2)$ & $136(95,8)$ & \multirow{2}{*}{0,676} \\
\hline Não & $1(1,7)$ & $59(98,3)$ & \\
\hline \multicolumn{4}{|l|}{ Diagnóstico Psiquiátrico* } \\
\hline $\operatorname{Sim}$ & $3(3,8)$ & $75(96)$ & \multirow{2}{*}{0,705} \\
\hline Não & $5(4,3)$ & $112(95,7)$ & \\
\hline
\end{tabular}

Notas: ${ }^{*} \mathrm{n}(\%) ;$ "mediana (intervalos interquartílicos).

${ }^{* *} I M C:$ índice de massa corporal.

Fonte: banco de dados dos autores

Afastamentos superiores a 15 dias foram estatisticamente associados à ocupação de auxiliar e técnico de enfermagem $(\mathrm{p}=0,016)$, a maior escolaridade $(\mathrm{p}=0,035)$ e menor mediana de idade $(\mathrm{p}=0,006)$. Acrescido a esta análise, quando vislumbrado o tempo de 30 dias de afastamento, foram observados que trabalhadores com maiores medianas no peso estiveram significativamente associados ao tempo de afastamento $(\mathrm{p}=0,043)$. 
No gráfico 1 é possível encontrar a frequência de afastamentos no período de 2012 a 2017 dos indivíduos da amostra conforme repetições nos afastamentos.

Gráfico 1 - Frequência de afastamentos por distúrbios osteomusculares no período de 2012 a 2017. Porto Alegre, RS. 2018.

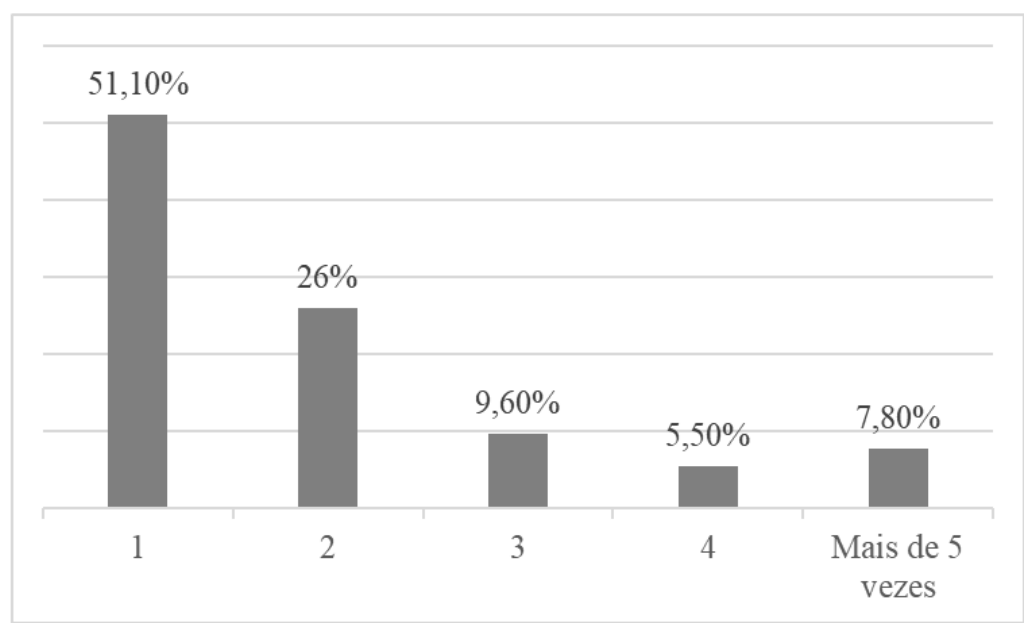

Os afastamentos por distúrbios osteomusculares conforme o CID foram por dorsalgia (41,5\%), lesões do ombro (25\%), transtorno de discos cervicais $(7,8 \%)$, sinovite e tenossinovite $(6,3 \%)$, entesopatia $(6 \%)$, transtorno em discos com radiculopatia $(5,1 \%)$, transtorno em discos intervertebrais $(2,9 \%)$, fascites $(2,5 \%)$ e outros $(2,9 \%)$.

É importante mencionar que na categoria Dorsalgia estão inseridas outros tantos CID’s que foram considerados na mesma classificação para um melhor entendimento e separação dos dados. Dorsalgias engloba Cervicalgias, Dores no Ciático, Lumbago com Ciática, Dor Lombar Baixa e Dorsalgia não especificada. Na categoria “Outros” estão inseridos Dor Articular, Síndrome Cervicobraquial e Outros Cistos de Bolsa Sinovial que apresentaram números reduzidos.

\section{Discussão}

O presente estudo verificou que 220 profissionais da enfermagem se afastaram por DORT totalizando 449 afastamentos, um percentual de $16,26 \%$ do total de afastamentos. O percentual encontrado é inferior ao que foi identificado em um estudo realizado em hospitais universitários de São Paulo, com 31\% de afastamentos por DORT. ${ }^{14}$ Entretanto, ainda está acima dos resultados de 
outras instituições, como um outro hospital universitário da região sul, o qual identificou que 12,5\% de afastamentos pelo mesmo motivo. ${ }^{15}$ Esta diferença sugere que características do local de trabalho podem minimizar o adoecimento da equipe de enfermagem por DORT e que há a necessidade de melhorias nas condições de trabalho da população estudada.

Não foram encontradas diferenças na distribuição do sexo e os tempos de afastamento por DORT. No entanto, estudo que visou estabelecer diferenças do sexo e a ocorrência de DORT em extremidades superiores com amostra de 1.177 indivíduos concluiu que as mulheres apresentam maior ocorrência desse agravo. ${ }^{16}$

Outra pesquisa brasileira com trabalhadores de enfermagem encontrou associações entre o sexo masculino e dor nos cotovelos $(\mathrm{RP}=5,5$, IC 95\%:1,1;25,5, $\mathrm{p}=0,028)$ e tornozelos $(\mathrm{RP}=5,1, \mathrm{IC}$ 95\%:1,3;19,2, $\mathrm{p}=0,016)$, e dor e inatividade física para os segmentos em cotovelos $(\mathrm{RP}=3,4, \mathrm{IC}$ 95\%:1,1;10,3, p=0,027) e joelhos ( $\mathrm{RP}=2,4, \mathrm{IC} 95 \%: 1,1 ; 5,0, \mathrm{p}=0,021)$. O mesmo estudo não encontrou diferença significativa para índice de massa corporal. ${ }^{17}$

Diferente dos resultados deste estudo quanto ao estado civil dos trabalhadores, estudo que avaliou 60.202 brasileiros encontrou menor chance de diagnóstico de DORT o fato de não ter parceiro $(\mathrm{IC} 95 \% 0,37-0,71){ }^{18}$

Auxiliares e técnicos de enfermagem foram os mais acometidos por DORT, sendo que 193 (98\%) dos profissionais de nível médio ficaram mais de 15 dias afastados. Entende-se que este dado está relacionado às atividades desenvolvidas por esta categoria profissional, visto que os auxiliares e técnicos possuem atribuições que demandam grande esforço físico para a realização dos cuidados em pacientes dependentes. Além disso, questões relacionadas à ergonomia, condições de trabalho, quadro de pessoal e sobrecarga de trabalho devem ser consideradas. Estudo realizado em uma unidade de internação demonstrou que o incremento de 40,0\% no número de enfermeiros e 16,0\% no número de técnicos de enfermagem resultou na redução de $12,0 \%$ no percentual de afastamentos por doença. ${ }^{19}$ 
11 | Souza YMN, Dal Pai D, Junqueira LM, Macedo ABT, Tavares JT, Chaves EBM

Verificou-se que os profissionais afastados possuíam idade média de 48 anos, com idade mínima de 28 anos, e houve diferença estatisticamente significativa $(\mathrm{p}=0,006)$ quando comparada à idade dos profissionais que se afastaram mais de 15 dias com os que se afastaram por tempo menor, demonstrando que as lesões osteomusculares acometem profissionais mais jovens, em fase produtiva. Em outra pesquisa também se identificou maior tempo de afastamento em trabalhadores com menor tempo de ocupação na instituição. ${ }^{20}$

Uma avaliação realizada em Pronto Atendimento brasileiro revelou que a maioria dos trabalhadores afastados $(48,6 \%)$ tinha menos de um ano de serviço na instituição. ${ }^{21}$ Entretanto, outra pesquisa realizada com trabalhadores de diversos setores demonstrou que o adoecimento apareceu em profissionais com maior tempo de serviço. ${ }^{15}$

Quanto ao achado que versa sobre a escolaridade, não foram encontrados dados na literatura. Trata-se de aspecto a ser mais explorado, considerando que os profissionais de enfermagem de nível médio costumam realizar atividades de maior esforço físico quando comparado aos profissionais de nível superior, como empurrar macas, fazer mudança de decúbito, banho no leito, dentre outros.

Sobre a localização da dor, os dados encontrados convergem com o que há na literatura nacional e internacional. Avaliação realizada na Estônia demonstrou que $57 \%$ dos profissionais pesquisados possuíam dor na região lombar e $56 \%$ no pescoço. ${ }^{8}$ Uma revisão da literatura com amostra de 132 artigos apontou que as regiões prevalentes em que os indivíduos da enfermagem relatam dores musculoesqueléticas são região lombar (93\%), pescoço (47\%) e ombros $(46 \%) .{ }^{22} \mathrm{O}$ principal fator desencadeante em profissionais da enfermagem, como também de piora da lombalgia, é a manipulação do paciente. Equipamentos para mobilização de indivíduos dependentes e apoio aos trabalhadores por meio de tratamentos não farmacológicos podem prevenir lesões da região lombar e minimizar os sintomas. ${ }^{20}$ 
Embora ainda se tenha dificuldade em identificar o que é causa e o que se refere ao efeito, já se sabe que existe uma ligação entre transtornos mentais e doenças osteomusculares em profissionais da enfermagem..$^{23}$ Nesta investigação 78 trabalhadores afastados também apresentavam diagnóstico psiquiátrico, dentre eles, 33 com depressão. Acerca disso, estudo apontou que são fatores associados a uma maior chance de se ter DORT o diagnóstico de depressão (IC95\% 0,37 - 0,71) e de artrite e reumatismo (IC95\% 1,68 - 3,44). ${ }^{18}$

Assim, fatores psicossociais e problemas de saúde mental, principalmente sintomas de estresse somático parecem ter impacto importante para a presença de dor musculoesquelética. ${ }^{24}$ Estudo brasileiro que buscou associar dor crônica musculoesquelética com distúrbios psiquiátricos encontrou que a dor é fator de risco independente para distúrbios psiquiátricos e risco de suicídio, o que indica a necessidade de uma abordagem multidisciplinar para o problema. ${ }^{25}$

Em hospitais universitários de São Paulo verificou-se que as notificações de adoecimento relacionadas a transtornos mentais e comportamentais já são a segunda causa de adoecimento na enfermagem, com 23,2\%. ${ }^{14} \mathrm{Na}$ Europa, uma pesquisa que objetivou mostrar a prevalência de dores musculoesqueléticas na equipe de enfermagem identificou forte relação com estresse, burnout e sintomas depressivos. ${ }^{24}$ Os profissionais atribuem os sintomas psicossociais à imposição de tempo para a realização das tarefas, gerando aceleração dos movimentos do corpo e postura inadequada, fatores relevantes para o surgimento de dor e desconforto musculoesqueléticos. ${ }^{19}$

Identificou-se que o serviço que mais apresentou afastamentos foi de internação clínica, junto ao qual internam doentes crônicos, com nível de dependência elevada para os cuidados de enfermagem, possuindo assim um tempo maior de permanência hospitalar. Acerca disso, inferese a importância de estratégias equitativas na distribuição de pessoal, acompanhando a divisão do trabalho nas tarefas e escalas, a fim de evitar a sobrecarga física e psicológica que impacta sobre o trabalhador. 
13 | Souza YMN, Dal Pai D, Junqueira LM, Macedo ABT, Tavares JT, Chaves EBM

Verificou-se que $48,9 \%$ apresentaram mais de um afastamento no período estudado, o que demonstra a persistência do agravo ou as co-morbidades do trabalhador. Acerca disso, uma pesquisa identificou que o agravamento da doença pode ocorrer por acomodação dos trabalhadores em relação aos sintomas osteomusculares, fato que muitas vezes ocasiona demora na procura dos serviços de saúde e o diagnóstico da condição de doença, seja por falta de tempo ou por dificuldade de acesso. ${ }^{19}$

Embora o percentual de afastamentos por DORT pareça pouco $(9,94 \%)$ em um primeiro olhar, torna-se imprescindível pensar na magnitude do problema, fato que se expressa nos demais resultados. O acometimento da saúde do trabalhador da enfermagem causa sofrimento psíquico e dificulta a realização de atividades do cotidiano, visto que a dor restringe ou impossibilita ações simples como subir escadas, caminhar, ficar em pé, dormir, entre outros. Além dos problemas citados acima, deve-se salientar o impacto econômico dessa modalidade de adoecimento dos trabalhadores e, considerando a sua expressiva importância no contexto da enfermagem, são prementes ações resolutivas.

\section{Conclusão}

Este estudo teve o objetivo de descrever características sociodemográficas e ocupacionais de trabalhadores da equipe de enfermagem afastados por distúrbios osteomusculares em hospital universitário e sua associação com o tempo de afastamento. Os resultados encontrados evidenciam a necessidade de viabilizar o acesso às tecnologias que minimizem o excessivo e frequente esforço físico e posturas prejudiciais adotadas na realização de atividades de assistência em enfermagem, especialmente entre auxiliares e técnicos de enfermagem, associadas à adesão às medidas preventivas por parte dos trabalhadores. Além disso, destacamse as condições de trabalho como aspecto de direta interferência sobre as cargas de trabalho impróprias à manutenção da integridade física e psicológica do trabalhador de enfermagem. 
Sugere-se também o investimento em estudos que avaliem a dor osteomuscular em associação com as condições de trabalho. O efeito das terapias não farmacológicas como medida de prevenção ao aparecimento de lesões osteomusculares.

Como limitações do estudo estão os registros por vezes incompletos, a impossibilidade de avaliar a efetividade física dos trabalhadores afastados e o desenho transversal, que impossibilita inferências relacionadas à causalidade dos agravos que levam ao afastamento.

\section{Referências}

1. Lida I, Guimarães LBM. Ergonomia: projeto e produção. São Paulo (SP): Blucher; 2016.

2. Sousa BVN, Silva DLS, Ferreira MS, Santana RR, Cunha WC, Brito CO. Lesões por esforço repetitivo em profissionais de enfermagem: revisão sistemática. Rev Bras Saúde Func [Internet]. 2016 [acesso em 2019 jan 23];1(3). Disponível http://www.seeradventista.com.br/ojs/index.php/RBSF/article/view/758/646

3. Shoji S, Souza NVDO, Farias SNP. Impacto do ambiente laboral no processo saúde doença dos trabalhadores de enfermagem de uma unidade ambulatorial especializada. REME Rev Min Enferm [Internet]. 2015 [acesso em 2018 jun 20];19(1):43-8. Disponível em: http://www.reme.org.br/artigo/detalhes/984 doi:10.5935/1415-2762.20150004

4. Lourenção LG, Sanches NF, Todesco TN, Soler ZASG. Queixas de distúrbios osteomusculares em aprimorandos e aperfeiçoandos atuantes em um hospital de ensino. Rev Enferm UFPE On Line [Internet]. 2017 [acesso em 2017 jul 07];11(1):383-92. Disponível em: https://periodicos.ufpe.br/revistas/revistaenfermagem/article/view/11919/14412 doi:10.5205/1981-8963v11i1a11919p383-392-2017

5. Paula AA, Sanchez MCO, Pereira MJ. Lesões por esforço repetitivo/distúrbio osteomuscular relacionado ao trabalho: contribuições para a prevenção em trabalhadores de enfermagem. Acc Cietna (Chiclayo) [Internet]. 2016 [acesso em 2017 jul 07];3(2):11. Disponível em: http://revistas.usat.edu.pe/index.php/cietna/article/view/42

6. Ferreira JM. Relação dos fatores de risco ligados ao trabalho interativo usando computador e telefone com a etiopatogenia dos distúrbios osteomusculares de região cervical, ombros e membros superiores [tese]. São Paulo: Universidade de São Paulo; 1997. 109 p.

7. Vidor CR, Mahmud MAI, Farias LF, Silva CA, Ferrari JN, Comel JC, et al. Prevalência de dor osteomuscular em profissionais de enfermagem de equipes de cirurgia em um hospital universitário. 
Acta Fisiátrica [Internet]. 2014 [acesso em 2017 jun 09];21(1):6-10. Disponível em: http://www.revistas.usp.br/actafisiatrica/article/view/103819/102290 doi:10.5935/0104-7795.20140002

8. Merisalu E, Animägi L, Oha K, Freimann T, Sirk T. Job-specific factors and prevalence of multiple and disabling musculoskeletal pain among office workers, nurses and caregivers in Estonia. Proc Latv Acad Sci B [Internet]. 2016 [acesso em 2017 jun 20];70(5):286-93. Disponível em: https://content.sciendo.com/view/journals/prolas/70/5/article-p286.xml doi:https://doi.org/10.1515/prolas2016-0044

9. Arvidsson I, Simonsen JG, Dahlqvist C, Axmon A, Karlson B, Björk J, et al. Cross-sectional associations between occupational factors and musculoskeletal pain in women teachers, nurses and sonographers. BMC Musculoskeletal Disord [Internet]. 2016 [acesso em 2017 jun 20];17(35):1-15. Disponível em: https://www.ncbi.nlm.nih.gov/pmc/articles/PMC4717636/ doi: 10.1186/s12891-016-0883-4

10. Akbari H, Akbari H, Gholami-Fesharaki M, Hosseinabadi, MB. Assessing the risk of manual handling of patients and its relationship with the prevalence of musculoskeletal disorders among nursing staff: Performance evaluation of the MAPO and PTAI methods. Iran Red Crescent Med J [Internet]. 2017 [acesso em 2017 jun 20];19(2):1-8. Disponível em: https://www.researchgate.net/publication/307899285_Assessing_the_Risk_of_Manual_Handling_of_Patie nts_and_Its_Relationship_with_the_Prevalence_of_Musculoskeletal_Disorders_Among_Nursing_Staff_ Performance_Evaluation_of_the_MAPO_and_PTAI_Methods

12. Marques D. O absenteísmo-doença da equipe de enfermagem de um hospital universitário [dissertação]. Goiânia: Universidade Federal de Goiás; 2014.

13. Santos LSF, Hansel CG, Camacho ACLF, Oliveira BGRB, Shiraishi R. Evidências de absenteísmo na enfermagem: revisão integrativa. Rev Enferm UFPE On Line [Internet]. 2014 [acesso em 2017 jul 19];8(10): 3483-91. Disponível em: https://periodicos.ufpe.br/revistas/revistaenfermagem/article/view/10081/10526 doi:10.5205/reuol.6039-55477-1-ED.081020128

14. Guimaraes ALO, Felli VEA. Notificação de problemas de saúde em trabalhadores de enfermagem de hospitais universitário. Rev Bras Enferm [Internet]. 2016 [acesso em 2018 jun 11];69(3):507-14. Disponível em:http://www.scielo.br/pdf/reben/v69n3/0034-7167-reben-69-03-0507.pdf

doi:http://dx.doi.org/10.1590/0034-7167.2016690313i

15. Prestes FC. Absenteísmo-doença em trabalhadores de enfermagem de um hospital universitário: estudo de métodos mistos [tese]. Santa Maria: Universidade Federal de Santa Maria; 2017. 199 p.

16. Almeida CGSTG, Fernandes RCP. Distúrbios músculoesqueléticos em extremidades superiores distais entre homens e mulheres: resultados de estudo na indústria. Rev Bras Saúde Ocup [Internet]. 2017 [acesso em 2019 nov 15];42:e3. Disponível em: http://www.scielo.br/scielo.php?script=sci_arttext\&pid=S0303-76572017000100202\&lng=en doi:http://dx.doi.org/10.1590/2317-6369000125515 
17. Maciel Júnior EG, Trombini-Souza F, Maduro PA, Mesquita FOS, Silva TFA. Self-reported musculoskeletal disorders by the nursing team in a university hospital. BrJP [Internet]. 2019 jun [acesso em 2019 nov 18];2(2):155-8. Disponível em: http://www.scielo.br/scielo.php?script=sci_arttext\&pid=S259531922019000200155\&lng=en doi: http://dx.doi.org/10.5935/2595-0118.20190028

18. Assunção AA, Abreu MNS. Fatores associados a distúrbios osteomusculares relacionados ao trabalho autorreferidos em adultos brasileiros. Rev Saúde Pública. 2017;51 Supl 1:10s.

19. Oliveira VC, Almeida RJ. Aspectos que determinam as doenças osteomusculares em profissionais de enfermagem e seus impactos psicossociais. J Health Sci [Internet]. 2017 [acesso em 2018 jun 20];19(2):1305. Disponível em: http://www.pgsskroton.com.br/seer/index.php/JHealthSci/article/view/4272/3565

20. Quadros DV, Magalhães AMM, Mantovani VM, Rosa DS, Echer IC. Análise de indicadores gerenciais e assistenciais após adequação de pessoal de enfermagem. Rev Bras Enferm [Internet]. 2016 ago [acesso em 2019 out 31];69(4):684-90. Disponível em: http://www.scielo.br/scielo.php?script=sci_arttext\&pid=S0034-71672016000400684\&lng=pt doi:http://dx.doi.org/10.1590/0034-7167.2016690410i

21. Silva RF, Silva SF, Almeida NM, Barbosa TC, Quaresma FRP, Maciel ES. Presença de distúrbios osteomusculares em enfermeiros de unidades de pronto atendimento. Rev Enferm Atenção Saúde. [Internet]. 2017 [acesso em 2018 jun 15];6(2):2-11. Disponível em: http://seer.uftm.edu.br/revistaeletronica/index.php/enfer/article/view/2081/pdf

22. Davis KG, Kotowski SE. Prevalence of musculoskeletal disorders for nurses in hospitals, long-term care facilities, and home health care: a comprehensive review. Human Factors [Internet]. 2015 [acesso em 2018 jun 04];57:754-92. Disponível em: https://www.ncbi.nlm.nih.gov/pubmed/25899249 doi:10.1177/0018720815581933

23. Borges TP, Kurebayashi LFS, Silva MJP. Occupational low back pain in nursing workers: massage versus pain. Rev Esc Enferm USP [Internet]. 2014 ago [acesso em 2019 out 31];48(4):670-6. Disponível em: http://www.scielo.br/scielo.php?script=sci_arttext\&pid=S0080-62342014000400670\&lng=en doi:http://dx.doi.org/10.1590/S0080-623420140000400014

24. Freimann T, Pääsuke M, Merisalu E. Work-related psychosocial factors and mental health problems associated with musculoskeletal pain in nurses: a cross-sectional study. Pain Res Manag [Internet]. 2016 [acesso em 2018 jun 04];2016:9361016. Disponível em: https:/www.ncbi.nlm.nih.gov/pubmed/27885319 doi:10.1155/2016/9361016

25. Duque RH, Campos VR, Campanha JA, Cristo VV, Andrade CVC, Gavi MBRO. Dor crônica musculoesquelética é fator de risco para distúrbios psiquiátricos e suicídio. Rev Bras Reumatol [Internet]. 2017 [acesso em 2019 nov 2019];57(Supl 1):S405-S406. Disponível em: https://www.sciencedirect.com/science/article/pii/S0482500417308549 


\section{Autor correspondente}

Yuri Marques de Souza

E-mail: yurims43@hotmail.com

Endreço: rua alcebíades Azeredo dos Santos, 111, Sítio São José - Viamão

CEP: 94410200

\section{Contribuições de Autoria}

\section{1 - Yuri Marques Souza}

Concepção e planejamento do projeto de pesquisa, obtenção, análise e interpretação dos dados, redação e revisão crítica.

\section{2 - Daiane Dal Pai}

Concepção e planejamento do projeto de pesquisa, obtenção, análise e interpretação dos dados, redação e revisão crítica.

\section{3 - Larissa Martini Junqueira}

Interpretação dos dados, redação e revisão crítica.

\section{4 - Andréia Barcellos Teixeira Macedo}

Interpretação dos dados, redação e revisão crítica.

\section{5 -Juliana Petri Tavares}

Interpretação dos dados, redação e revisão crítica.

\section{6 - Eunice Beatriz Martin Chaves}

Interpretação dos dados, redação e revisão crítica.

\section{Como citar este artigo}

Souza YMN, Dal Pai D, Junqueira LM, Macedo ABT, Tavares JT, Chaves EBM. Caracterização dos trabalhadores da enfermagem afastados por distúrbios osteomusculares em hospital universitário. Rev. Enferm. UFSM. 2020 [Acesso em: Anos Mês Dia];vol.10 e10: 1-17. DOI:https://doi.org/10.5902/2179769236767 